\title{
Inaccurately measured poorer cognition as a result of an auditory deficit
}

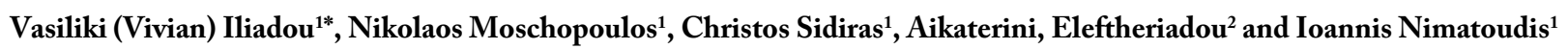 \\ ${ }^{1}$ Clinical Psychoacoustics Lab, 3rd Department of Psychiatry, Neuroscience Sector, Medical School, Aristotle University of Thessaloniki, Thessaloniki, Greece \\ ${ }^{2}$ Department of Occupational Therapy, Metropolitan College, Thessaloniki, Greece
}

\begin{abstract}
Background: Accuracy of measured auditory memory may be negatively impacted by non-proper functioning of the auditory system (i.e. hearing loss or auditory processing disorder).

Purpose: How important is this impact in clinical evaluation of short-term and working memory using the digit span test? The target group used is that of psychiatric patients as this patient population has both commonly observed cognitive deficits and undocumented hearing loss and auditory processing deficits.

Research design: Cross sectional.

Study Sample: Twenty-two psychiatric inpatients were evaluated for short-term and working memory.

Data collection and Analysis: The testing performed included (i) digit span evaluation at an intensity of 50dB HL, (ii) pure tone audiometry for hearing sensitivity evaluation and (iii) digit span evaluation at an intensity of 50dB SL (corrected based on the exact ear specific hearing sensitivity). Repeated measures ANOVA was implemented to test for differences between the two conditions (i \& iii).

Results: There was a documented improvement of short-term memory results due to adapting intensity of the digit span test according to each ear's specific hearing sensitivity as measured by pure tone audiometry. The effect $(\mathrm{F}=5.047, \mathrm{p}=.036)$ was present when evaluating the backwards digit span score.

Conclusions: Adapting digit span test implementation intensity will provide short-term and working memory results that are more accurate and avoid over-diagnosis of cognitive deficits. The paradigm of the present study is that of psychiatric disorders but there is no reason why this is not valid for any population.
\end{abstract}

Abbreviations: APD: Auditory Processing Disorder; dB: decibels; HL: Hearing Level; IQ: Intelligence Quotient; SL: Sensation Level.

\section{Introduction}

Cognition's complex interplay with hearing as well as with mental disorders is well established. Cognitive deficits are very commonly observed in psychiatric disorders. Emil Kraepelin, one of the most famous psychiatrists of all time, focused on the cognitive decline as a core symptom of schizophrenia. Consequently, he named the disorder "dementia praecox" [1]. During the last decades, a great number of publications have shed light on the nature and importance of cognitive impairment in schizophrenia, using the latest advances in science. Today, it is widely accepted that patients with schizophrenia have poorer performance across all known neuropsychological tests and domains compared to healthy individuals (Schaefer et al., 2013). Furthermore, cognitive impairment is considered a "core feature" of schizophrenia by some modern authors [2]. Kraepelin believed that cognitive decline wasn't a feature of bipolar disorder, but recent research data seem to contradict that view. An extensive review [3] confirmed the presence of cognitive deficits involving attention, executive function and verbal memory in all phases of bipolar disorder. A meta-analysis [4] reviewed the data that compared cognitive functioning across schizophrenia, schizoaffective disorder and affective psychosis (psychotic depression, psychotic bipolar disorder). In a number of cognitive domains, patients with schizophrenia performed more poorly than the other patients, but the overall differences were not statistically significant among the three groups.

Communication through speech perception requires both attention and memory cognitive skills to be effective. Short term memory is defined as the ability to accurately represent the auditory environment during short time periods of several seconds; often referred to as echoic memory [5]. Working memory is thought to be a more complex type of memory [6] involving auditory stimuli manipulation before output production. The most commonly used neuropsychological test of short-term and working memory is the Digit Span test [7]. The test requires the subject tested to repeat an increasing number of digits with an initiation of two digits; two trials with different digits are present at each increasing list length. Completion of the test is reached when the subject fails to correctly repeat one of the trials at each list length or when the test material is exhausted. The resulting score is computed as the addition of accurately reported digits. The test includes two parts;

${ }^{\star}$ Correspondence to: Vasiliki (Vivian) Iliadou, Clinical Psychoacoustics Lab, 3rd Department of Psychiatry, Neuroscience Sector, Medical School, Aristotle University of Thessaloniki, PC 54124 Thessaloniki, Greece, E-mail: viliad@auth.gr

Key words: auditory processing disorder, cognition, hearing, memory, measurement instruments in psychiatric care

Received: October 16, 2018; Accepted: October 25, 2018; Published: October 30,2018 
the first part including the forward repeated digits and the second part composed of the reversely reported digits. Each separate list is scored as one if the individual tested correctly reports it or zero if there is an error during reporting (either reporting a different digit or mixing the order of report). There is a total score including both forward and backward reports and a separate score for each of the forward and backward responses. Forward digits score is thought to be an index of short-term memory and attention, whereas backwards digits score is thought to reflect working memory and linked with executive functioning of the prefrontal cortex. Auditory short term memory is shown to be related with learning how to read with dyslexics exhibiting poor memory spans [6]. In addition, accomplished hard of hearing readers have better verbal memory than poor hearing impaired readers having similar non-verbal IQ $[6,8]$. In spite of the benefit of cochlear implantation in hearing and cognition, the existence of a decreased auditory memory in implanted children may be attributed to a continued auditory deprivation during childhood, as a consequence of hearing with a cochlear implant [6]. This indicates a causal relation between hearing and cognition with hearing deficits leading to cognitive ones but is still largely overlooked when investigating less profound hearing losses and/or auditory processing deficits.

Auditory memory is supposed to be an accurate representation of our auditory environment [9] but its accuracy may be decreased in cases when the auditory sensory system is not functioning properly. The etiologic for limited auditory functioning may be a hearing loss or an auditory processing disorder (APD). Although hearing loss is easier to diagnose than APD, usually psychiatric patients and their significant others acknowledge severe forms of hearing loss, while ignoring moderate or mild forms especially if unilaterally present (involving only one ear). How much impact might a hearing disorder (hearing loss \& auditory processing disorder) have on short-term memory evaluation is not known, specifically when considering psychiatric patients. Backwards digit span results are thought to be linked to verbal working memory through re-ordering requirements of mnemonic verbal information. As this is a more complicated task than forward digit span it is thought to reflect prefrontal cortex functioning and include executive function as well as simple temporal storage of auditory information [10]. Sensory information is gathered from the prefrontal cortex for actions (execution) to take place. As there is no direct connection between the prefrontal cortex and the auditory peripheral system, auditory information is conveyed through other cortical or sub cortical structures [11] and these pathways functional integrity might be essential for executive functioning.

Cognition is very often evaluated through the auditory modality using verbal auditory stimuli. This practice leads to permitting cognitive measurements to be negatively influenced (downgraded) in case of any pathology interfering with hearing ability. In cases of severe hearing loss, also known as deafness, it is much easier to tap into the correlation with low cognition. Unfortunately, cases of moderate, mild or unilateral hearing loss as well as auditory processing disorder may be in most cases overlooked and the individual tested has the possibility of being assigned a lower cognitive skill than he/she actually has. In order to assess if this hypothesis is true we tested psychiatric inpatients. Evaluation included a digit span test for short-term memory evaluation presented at an intensity of 50dB HL (not taking account of the exact hearing sensitivity of the patient) and a repetition of the same test at an intensity of $50 \mathrm{~dB} \mathrm{HL}+$ average hearing threshold of tested frequencies during pure tone audiometry. The digit span test was administered through a GSI 61 clinical audiometer and intensity was adjusted to fit the exact average hearing threshold for each ear even though presentation was binaural (presented simultaneously to both ears). Our hypothesis was that the two measurements of short-term memory would differ and the optimal result would be the one where hearing sensitivity of each tested patient was taken into account [12]. If this hypothesis is confirmed, then clinical implications include careful consideration of testing environment and speech intensity selected during the digit span test which is clinically administered by clinical psychologists as well as audiology referrals for hearing threshold of each patient to be assessed at least in cases of abnormal short-term memory. The psychiatric population used in the present study was elected due to high occurrence of cognitive deficits and high occurrence of undocumented hearing loss as well as auditory processing deficits.

\section{Method}

Twenty-two psychiatric inpatients (14 females) being at a stable condition were included in this study. This study was carried out following written informed consent from all individuals tested. This is in accordance with the Declaration of Helsinki. The study was approved by the Ethics and Bioethics Committee of the Aristotle University of Thessaloniki. Mean age of the individuals tested was 40.3 (sd=12), formal education was 12.4 years as a mean $(\mathrm{sd}=2.8)$, while specific music education was present in this patient group (mean=0.7, $\mathrm{sd}=1.7$ ). Schizophrenia was the diagnosis for 12 patients, brief psychotic disorder for 1, schizoaffective disorder for 4 and bipolar disorder for 5 patients. Otoscopy was performed in all patients and in four cases when cerum was present it was removed before testing for short-term memory and audiometry. In all other cases otoscopy results were unremarkable. The testing performed included (i) digit span evaluation at an intensity of $50 \mathrm{~dB} \mathrm{HL}$, (ii) pure tone audiometry for hearing sensitivity evaluation and (iii) digit span evaluation at an intensity of 50dB SL (corrected based on the exact ear specific hearing sensitivity). To minimise learning effects being interpreted as a result of difference in hearing sensitivity during implementation of the digit span test, half of the patients were tested in the sequence of i-ii-iii and half of them ii-iii-i. The second measurement of the digit span test took place 7-10 days following the first evaluation. All testing took place in a sound treated booth with the examiner being outside the booth while communicating verbally through a GSI-61 audiometer. Patient and examiner had visual contact as well through a window. Pure tone audiometry was performed using a GSI-61 audiometer calibrated per standard guidelines [5]. The other two measurements included the digit span test at one intensity level (50dB HL) that is thought to be approximating typical everyday speech without any consideration on patient's actual hearing sensitivity and at another at an intensity level of 50dB SL taking into account the ear specific hearing sensitivity of each patient tested. The patient was asked to repeat numbers as they were being heard, in the exact same order for the first part of the test and in a backward order for the second part of the test. Repetition was initiated following the completion of all digits included in each item. Before each item a $1000 \mathrm{~Hz}$ pure tone of $500 \mathrm{msec}$ duration was included to attract and maintain attention throughout the test. The resulting overall score of the digit span test is the addition of the forward digit span test score and the backward digit span test score. Scores for each of the two conditions of the digit span test are calculated attributing one point to each correctly repeated series of numbers and zero point when the numbers are incorrectly repeated (either in terms of order or actual number repeated). The test initiates with two items including two digits and each of the items may be attributing scores of one or zero according to correct or incorrect 
recognition by the individual tested. Consequently, two items of three digits are heard and scored in the same way and the test proceeds with longer series of numbers until it reaches the end with two items of nine digits each or until the individual tested incorrectly repeats two items of the same length (in terms of number of digits). The forward digit span resulting score is computed by adding scores for each item tested. Then the individual tested is instructed to repeat digits in a reverse manner. The subtest starts with two items of two digits and proceeds adding one more digit for items of the same length until it reaches eight digits length items or until the individual tested repeats incorrectly two items of the same digit length. The scoring used in this study is the one proposed both as a stand-alone short-term memory evaluation and when used in IQ evaluation testing but given that recent research [13] uses the reliable digit span scoring calculations were performed for this method as well for comparison purposes. This measurement does not take into account the correct repeat of digits of either forwards or backwards digits when one of the same digits trials is wrong. An individual has to correctly identify both same digit trials at each level of increasing digits to be able to add one correct answer to his scoring.

\section{Statical analysis}

Statistical analysis was implemented using the SPSS version 23. Results followed a normal distribution under the criterion of skewness and kurtosis $\mathrm{z}$ values ranging between -1.96 and $1.96[1,14]$ hence parametric tests were used for statistical analysis. Repeated measures ANOVA was implemented to test for differences between the two evaluation conditions, one at an intensity of typical everyday speech (possibly mirroring psychologist's implementation method) and the other adjusting intensity of uttered series of digits to ear specific hearing threshold (as measured by pure tone audiometry).

\section{Results}

There was a documented improvement of short-term memory results (controlling for learning effects) due to adapting intensity of the digit span test according to each ear's specific hearing sensitivity as measured by pure tone audiometry (Figure 1a). The effect was present when evaluating the backwards digit span score (Figure 2) but not when evaluating the forward digit span score. Specifically, repeated measures ANOVA showed no significant difference between the two conditions (with and without taking into account ear specific hearing threshold) of forward presentation $(\mathrm{F}=.522, \mathrm{p}>.05)$. The same analysis showed significant difference for backward presentation $(\mathrm{F}=5.047$, $\mathrm{p}=.036$ ). Correlations between the two different conditions reveal good correlation between forward Digit Span different intensity conditions and no correlation between backwards Digit Span different intensity conditions (Table 1). A 2 by 2 repeated measures ANOVA factors: a) level ( $50 \mathrm{~dB}$ vs $50 \mathrm{~dB}+\mathrm{PTA}$ ) and b) direction (forward vs backward) revealed significant effect of direction $(\mathrm{F}=82.1, \mathrm{p}<.001)$, forward scores being higher than backward ones, and interaction of level and direction $(\mathrm{F}=4.81, \mathrm{p}=.040)$. That is, level of presentation had a significant larger effect for backward digit span repetition than for forward repetition. ANOVA was executed for all measures (PTA both ears, DD scores forward and backward, 50dB and 50+PTA) to assess differences between men and women. PTA was not significantly different. Men scored better than women for backward in the $50 \mathrm{~dB}$ HL condition (mean $=4.86$ (1.17) vs mean=3.38 (.74) for men and women respectively). The remaining measures (i.e. total digit span score) were not different

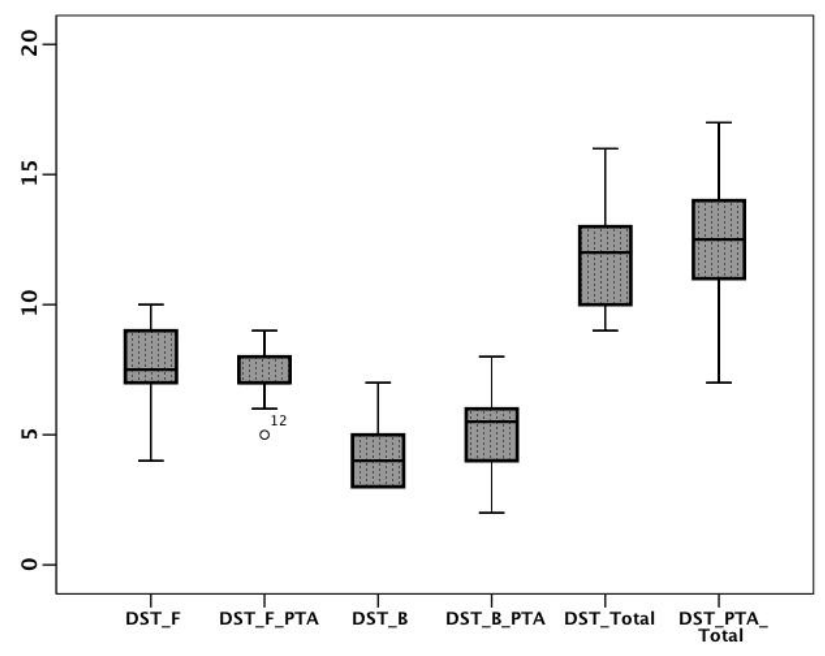

Figure 1A. Better short-term memory results due to adapting intensity of the digit span test according to each ear's specific hearing sensitivity as measured by pure tone audiometry

DST_F: Forward Digit Span Test result administered at 50dB HL (approximating typical everyday speech intensity).

DST_F_PTA: Forward Digit Span Test result administered at 50dB HL plus average hearing threshold as measured by the pure tone audiogram for each ear (considering ear specific hearing sensitivity).

DST_B: Backwards Digit Span Test result administered at 50dB HL (approximating typical everyday speech intensity).

DST_B_PTA: Backwards Digit Span Test result administered at 50dB HL plus average hearing threshold as measured by the pure tone audiogram for each ear (taking into account ear specific hearing sensitivity).

DST_Total: Total Digit Span Test result (DST_F+DST_B) administered at 50dB HL (approximating typical everyday speech intensity).

DST_PTA Total: Total Digit Span Test result (DST F+DST B) administered at 50dB HL plus average hearing threshold as measured by the pure tone audiogram for each ear (taking into account ear specific hearing sensitivity).

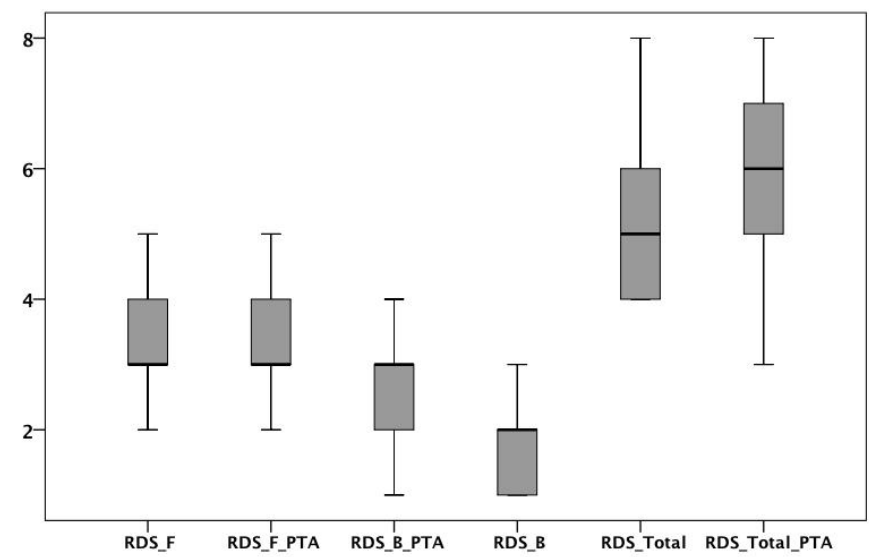

Figure 1B. All Digit Span scores calculated using the reliable digit span consideration approach for comparison with other studies. The difference is still evident as well as statistically significant for backwards reliable digit span score with and without controlling for hearing sensitivity (i.e. Pure Tone Audiometry)

RDS_F: Forward Reliable Digit Span,

RDS_F_PTA: Forward Reliable Digit Span controlling for Pure Tone Audiometry RDS_B_PTA: Backwards Reliable Digit Span controlling for Pure Tone Audiometry RDS_B: Backwards Reliable Digit Span

RDS_Total: Total Reliable Digit Span

RDS_Total_PTA: Total Reliable Digit Span controlling for Pure Tone Audiometry.

between the two conditions. The results (Figure 1b) stay the same when reliable digit span scoring is implemented with backwards digit span having lower values when hearing sensitivity is not considered for administration of appropriate/individualized intensity. 


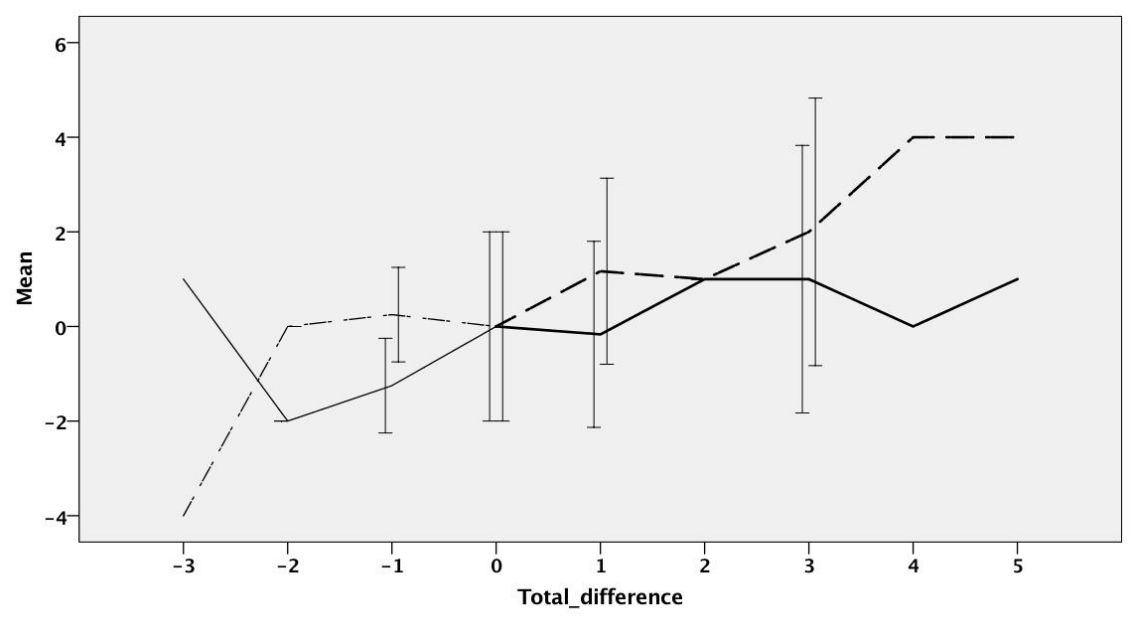

Figure 2. Backwards Digit Span is greater influenced by controlling for ear specific hearing threshold (PTA=Pure Tone Audiometry) than Forward Digit Span

Dashed line represents the difference between controlling for Backwards Digit Span with PTA minus not controlling for PTA. Continuous line represents the difference between controlling for Forward Digit Span and not controlling for PTA. Total_difference in the x axis represents the difference in Total Digit Span Test between controlling and not controlling for ear specific hearing threshold. Error bars of $2 \mathrm{sd}$ from the mean are visible in the figure for forward and backward digit span conditions

Table 1. Backwards Digit Span is not correlating between the two implementing conditions (controlling and not controlling for hearing sensitivity)

\begin{tabular}{|c|c|c|c|c|c|c|}
\hline & DST_F & DST_B & DST_F_PTA & DST_B_PTA & DST_Total & DST_PTA_Total \\
\hline DST_F r sig. & & $\begin{array}{l}0.031 \\
0.892\end{array}$ & $\begin{array}{c}602 * * \\
0.03\end{array}$ & $\begin{array}{l}0.350 \\
0.110\end{array}$ & $\begin{array}{c}0.771^{* *} \\
0.000\end{array}$ & $\begin{array}{c}0.529^{*} \\
0.011\end{array}$ \\
\hline DST_B r sig. & $\begin{array}{l}0.031 \\
0.892\end{array}$ & & $\begin{array}{c}-0.059 \\
0.795\end{array}$ & $\begin{array}{l}0.360 \\
0.100\end{array}$ & $\begin{array}{c}0.661^{* *} \\
0.001\end{array}$ & $\begin{array}{l}0.244 \\
0.273\end{array}$ \\
\hline DST_F_PTA r sig. & $\begin{array}{c}0.602 * * \\
0.003\end{array}$ & $\begin{array}{c}-0.059 \\
0.795\end{array}$ & & $\begin{array}{l}0.361 \\
0.099\end{array}$ & $\begin{array}{l}0.415 \\
0.055\end{array}$ & $\begin{array}{c}0.714^{* *} \\
0.000\end{array}$ \\
\hline DST_B_PTA r sig. & $\begin{array}{l}0.350 \\
0.110\end{array}$ & $\begin{array}{l}0.360 \\
0.100\end{array}$ & $\begin{array}{l}0.361 \\
0.099\end{array}$ & & $\begin{array}{c}0.429^{*} \\
0.020\end{array}$ & $\begin{array}{c}0.911 * * \\
0.000\end{array}$ \\
\hline DST_Total $r$ sig. & $\begin{array}{c}0.771 * * \\
0.000\end{array}$ & $\begin{array}{c}0.661^{* *} \\
0.001\end{array}$ & $\begin{array}{l}0.415 \\
0.055\end{array}$ & $\begin{array}{l}0.492 \\
0.020\end{array}$ & & $\begin{array}{c}0.553^{* *} \\
0.008\end{array}$ \\
\hline DST_PTA_Total rsig. & $\begin{array}{c}0.529 * \\
0.011\end{array}$ & $\begin{array}{l}0.244 \\
0.273\end{array}$ & $\begin{array}{c}0.714^{* *} \\
0.000\end{array}$ & $\begin{array}{c}0.911 * * \\
0.000\end{array}$ & $\begin{array}{c}0.553^{* *} \\
0.008\end{array}$ & \\
\hline
\end{tabular}

DST_F: Digit Span Forward,

DST_B: Digit Span Backwards

DST F PTA: Digit Span Forward controlling for PTA

DST_B PTA: Digit Span Backwards controlling for PTA,

DST_Total: Digit Span Total, DST_PTA_Total: Digit Span Total controlling for PTA

**Correlation is significant at the 0.01 level (2-tailed).

* Correlation is significant at the 0.05 level (2-tailed).

\section{Discussion}

\section{Controlling for hearing loss improves short-term memory scores}

Controlling for ear specific hearing threshold showed improvement of backward digit span scores that correspond to working memory, while forward and total digit span scores were not significantly influenced. Although there is evidence that elderly people with undiagnosed hearing loss may be inaccurately diagnosed with mild cognitive impairment [13], this is the first study showing influence of hearing on a measure of cognitive functioning in adults with a psychiatric disorder. Most of the widely used cognitive testing is implemented using the auditory modality and may be influence by any hearing deficit. In the vast majority of published research this is rarely acknowledged as it is assumed that using standardized cognitive tests is mirroring valid cognitive evaluation. However, standardization of cognitive tests is usually done in young adult populations that have lower possibilities of having an undiagnosed hearing impairment. This is partly accounted for when using age-corrected scale data [15]. This methodology is not providing specific individual data on hearing loss that may affect working memory as presbycusis does not develop with the same rate in each individual. The psychiatric clinical sample of the present study shows the possibility of having a mild to moderate hearing loss that the individual is unaware of to be as high as $45.5 \%$ and thus leading to lower short-term memory scores that are inaccurate. This might be tapping into everyday situations where people unaware of a mild to moderate hearing impairment may appear to be presenting with lower cognitive abilities than they might be capable of, provided management of the hearing impairment is ensured. In this sense a cognitive deficit may be the outcome of a hearing impairment.

\section{Backwards digit span score is negatively influenced by hearing impairment}

An interesting result of the present study is that the effect of lower short-term memory evaluation was present in backward digit span while absent in forward digit span test. This could be due to the interaction between cognitive load and listening effort in the context of limited cognitive reservoir. Listening effort is greater while trying to re-order (repeat series of digits in a backwards manner) than when trying to repeat in the exact same order and there is an added difficulty 
for the patients in the present clinical sample that have a mild to moderate hearing impairment. Although digit span test is thought to be measuring information storage and not processing [16], it appears that its backward score is influenced by an individual's ear specific hearing threshold. This might be linked to auditory perception and processing. Given that about half of the patients in our sample are at a disadvantage compared to the other half with normal hearing sensitivity, they seem to be allocating more cognitive resources to auditory perception and processing leaving them with less for storage and re-ordering of the verbal stimuli that they have to hear. The result is lower working memory scores that (i) may be correctly reporting cognitive issues experienced in everyday life but (ii) will be improved (or restored) if intensity of test administration is higher or hearing aids are implemented. Cognitive resources that are left unused for auditory perception and processing are indicative of the amount of information that can be transferred into long-term memory and consolidated as knowledge during learning [17]. With this knowledge, long-term untreated mild to moderate hearing impairment may lead to learning difficulties.

\section{Repeatability of results given by psychiatric patients}

Reliable measures of short-term and working memory may be difficult to obtain in psychiatric patients. In the present study implementation intensity of the digit span test differed between the two sessions in patients with mild to moderate hearing loss but were identical or similar in patients with normal hearing loss. Repeatability of these cases was high and in one case with the diagnosis of brief psychotic disorder the resulting digit span test was exactly the same in both sessions (Figure 3).

\section{What does backwards digit span measure?}

Backwards digits score is thought to reflect working memory and linked with executive functioning of the prefrontal cortex [10]. Working memory is perceived as a limited capacity system responsible for the processing and temporary storage of information. Since temporal manipulation (re-ordering) of the digits heard should take place in backwards digit test an executive function element is a prerequisite.

Limited capacity system: As working memory is defined as a limited capacity system when an individual is experiencing difficulty in listening due to a hearing impairment more capacity will be allocated to auditory perception and less will be available for information storage $[14,17]$. If this hearing impairment is addressed, either with the use of hearing aids or by increasing the voice intensity presentation of the digits during the test, then more capacity will be available for information storage. This mechanism may explain the present study's result of lower backward digit span scores when mild or moderate undiagnosed hearing loss is present and higher when this hearing loss is addressed by increasing the intensity according to the ear specific hearing loss. Short-term memory and auditory attention as measured by forwards digit span is found not to be influenced by mild to moderate hearing loss in the present study.

\section{Executive function}

Being able to re-order series of digits seems to be influenced by undiagnosed hearing loss during evaluation with digit span test and this is specifically depicted in the backwards digit span score. This should be considered when verbal working memory is considered impaired in schizophrenia patients using the digits span test. Results of the present study should be further confirmed using bigger patient samples and having adequate numbers per psychiatric diagnosis to evaluate possible differences between different psychopathologies.

\section{Awareness of hearing difficulties}

Self-report of hearing difficulties was not the case in the clinical sample of the present study. It might be argued that this is due to the presence of a psychiatric disorder. Awareness of difficulties may not be the same in different people depending on their personality, education, general health and mental state. It is often the case that hearing loss is perceived by the significant others. In the present study there was no suspicion of hearing loss possibly due to the general notion that hearing loss and deafness are identical. At a psychiatric general hospital setting the medical and health-related personnel is thinking about hearing loss when (i) a patient reports it, (ii) a caregiver reports it, or (iii) a patient does not seem to be responding consistently. In the last scenario it might not always be easy to interpret a patient's behaviour as a possible result of a hearing loss or a manifestation due to the psychiatric disorder. It is of interest that the degrees of hearing loss do not extend beyond moderate (up to $55 \mathrm{~dB} \mathrm{HL}$ ) in the present study. Patients and caregivers were unaware of the hearing loss and were possibly contributing any difficulties to the psychiatric disorder present or failing to report hearing difficulties as a minor issue compared to their main disorder. There is an indication that a brief questionnaire for
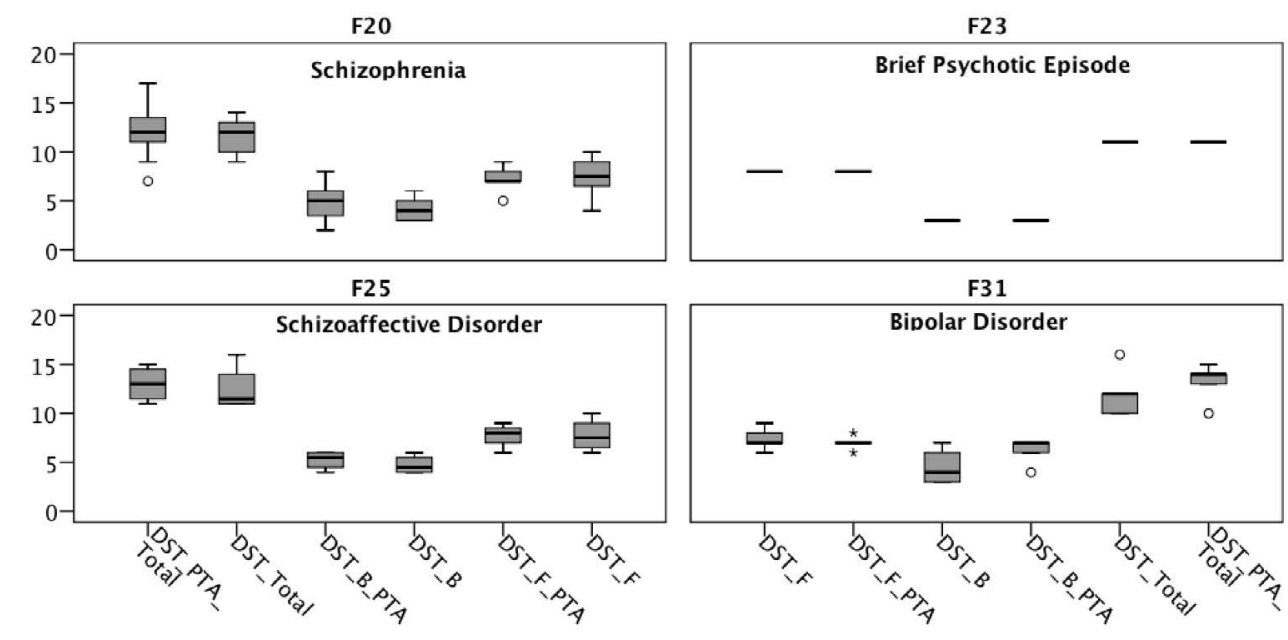

Figure 3. Results shown as a function of specific psychiatric diagnosis. Small number of cases for each diagnostic category do not permit for statistical analysis to be run in the present study. This figure is shown for repeatability of results in cases were normal hearing threshold results in same or very similar intensity level of digit span test administration (brief psychotic episode patient). A clear distinction between total digit span test taking into account ear specific hearing sensitivity vs failing to consider it, is shown for the bipolar disorder patients of this study 
hearing or a hearing sensitivity screening might be of benefit to these patients (i) in providing advice on how to deal with the problem and (ii) in ensuring more accurate cognitive testing.

\section{Conclusions and clinical implications}

Digit span testing as implemented in the everyday clinical practice may have many factors influencing results: voice intensity of the psychologist administering the test, rate of uttering the digits series in each item, clarity of voice (i.e. when having a cold) and mild to moderate hearing loss that may not be known to the tester or the individual being tested. In this sense cognitive measurements not acquired from 20-year olds (normative data are usually based on young adults, i.e. students) should be checked for their accuracy and ideally administered following a pure tone audiogram, so as to know hearing sensitivity of each individual tested. Adapting digit span test implementation intensity will provide short-term and working memory results that are more accurate and avoid over-diagnosis of cognitive deficits in psychiatric disorders. The psychiatric population used in the present study was elected due to high occurrence of cognitive deficits and high occurrence of undocumented hearing loss as well as auditory processing deficits. Awareness should be raised for careful interpretation of cognitive results in case hearing sensitivity or central auditory processing is not prior evaluated.

\section{References}

1. Kraepelin E (1919) Dementia Praecox and Paraphrenia. Livingstone, Edinburgh.

2. Elvevag B, Goldberg TE (2000) Cognitive impairment in schizophrenia is the core of the disorder. Crit Rev Neurobiol 14: 1-21 [Crossref]

3. Goldberg JF, Chengappa KNR (2009) Identifying and treating cognitive impairment in bipolar disorder. Bipolar Disord 11: 123-137. [Crossref]

4. Bora E, Yucel M, Pantelis C (2009) Cognitive functioning in schizophrenia, schizoaffective disorder and affective psychoses: Meta-analytic study. Br J Psychiatry 195: 475-482. [Crossref]
5. Jääskeläinen IP, Ahveninen J, Belliveau JW, Raij T, Sams M (2007). Short-term plasticity in auditory cognition. Trends Neurosci 30: 653-661. [Crossref]

6. Edwards L, Aitkenhead L, Langdon D (2016) The contribution of short-term memory capacity to reading ability in adolescents with cochlear implants. Int $J$ Pediatr Otorhinolaryngol 90: 37-42. [Crossref]

7. Woods DL, Kishiyama MM, Yund EW, Herron TJ, Edwards B, et al. (2011) Improving digit span assessment of short-term verbal memory. J Clin Exp Neuropsychol 33: 101111. [Crossref]

8. Hanson VL, Liberman IY, Shankweiler D (1984) Linguistic coding by deaf children in relation to beginning reading success. J Exp Child Psychol 37: 378-393. [Crossref]

9. Cramer D, Howitt D (2004) The SAGE Dictionary of Statistics. London: SAGE.

10. Werheid K, Hoppe C, Thöne A, Müller U, Müngersdorf M, et al. (2002) The adaptive digit ordering test: Clinical application, reliability, and validity of a verbal working memory test. Arch Clin Neuropsychol 17: 547-565. [Crossref]

11. Barbas H (2000) Connections underlying the synthesis of cognition, memory, and emotion in primate prefrontal cortices. Brain Res Bull 52: 319-330. [Crossref]

12. Iliadou V, Moschopoulos N, Sidiras C, Eleftheriadou A, Nimatoudis I (2018) Over-diagnosis of cognitive deficits in psychiatric patients may be the result of not controlling for hearing sensitivity and auditory processing. Psychiatry Clin Neurosci 79: 742. [Crossref]

13. Jorgensen LE, Palmer CV, Pratt S, Erickson KI, Moncrieff D (2016) The effect of decreased audibility on MMSE performance: A measure commonly used for diagnosing dementia. J Am Acad Audiol 27: 311-323. [Crossref]

14. PichoraFuller MK, Schneider BA, Daneman M (1995) How young and old adults listen to and remember speech in noise. J Acoust Soc Am 97: 593-608. [Crossref]

15. Babikian T, Boone KB, Lu P, Arnold G (2006) Sensitivity and specificity of various digit span scores in the detection of suspect effort. Clinical Neuropsychologist 20: 145159. [Crossref]

16. Daneman M, Merikle PM (1996) Working memory and language comprehension: A meta-analysis. Psychon Bull Rev 3: 422-433. [Crossref]

17. Pichora-Fuller MK, Kramer SE, Eckert MA, Edwards B, Hornsby BWY, et al. (2016) Hearing impairment and cognitive energy: The framework for understanding effortful listening (FUEL) Ear Hear 37: 5S-27S. [Crossref]

Copyright: ( 2018 Iliadou V. This is an open-access article distributed under the terms of the Creative Commons Attribution License, which permits unrestricted use, distribution, and reproduction in any medium, provided the original author and source are credited. 